\title{
Coupling of excitons and defect states in boron-nitride nanostructures
}

\author{
C. Attaccalite, ${ }^{1}$ M. Bockstedte, ${ }^{2,3}$ A. Marini, ${ }^{4,5,3}$ A. Rubio, ${ }^{3,6}$ and L. Wirtz ${ }^{7}$ \\ ${ }^{1}$ Institut Neel, CNRS/UJF, 25 rue des Martyrs BP 166, Bâtiment D, F-38042 Grenoble cedex 9 France \\ ${ }^{2}$ Lehrstuhl für Theoretische Festkörperphysik, Universität Erlangen-Nürnberg, Staudtstrasse 7 B2, D-91058 Erlangen, Germany \\ ${ }^{3}$ Nano-Bio Spectroscopy Group and ETSF Scientific Development Centre, Departamento Física de Materiales, Universidad del País Vasco, \\ Centro de Física de Materiales CSIC-UPV/EHU-MPC and DIPC, Av. Tolosa 72, E-20018 San Sebastián, Spain \\ ${ }^{4}$ Dipartimento di Fisica, Università di Roma “Tor Vergata," via della Ricerca Scientifica 1, I-00133 Roma, Italy \\ ${ }^{5}$ Ikerbasque, Basque Foundation for Science, E-48011 Bilbao, Spain \\ ${ }^{6}$ Fritz-Haber-Institut der Max-Planck-Gesellschaft, Berlin, Germany \\ ${ }^{7}$ Institute for Electronics, Microelectronics, and Nanotechnology (IEMN), CNRS-UMR 8520, Department ISEN, B.P. 60069, F-59652 \\ Villeneuve d'Ascq Cedex, France
}

(Received 3 January 2011; revised manuscript received 14 March 2011; published 29 April 2011)

\begin{abstract}
The signature of defects in the optical spectra of hexagonal boron nitride $(\mathrm{BN})$ is investigated using many-body perturbation theory. A single BN-sheet serves as a model for different layered BN nanostructures and crystals. In the sheet we embed prototypical defects such as a substitutional impurity, isolated boron and nitrogen vacancies, and the divacancy. Transitions between the deep defect levels and extended states produce characteristic excitation bands that should be responsible for the emission band around $4 \mathrm{eV}$, observed in luminescence experiments. In addition, defect bound excitons occur that are consistently treated in our $a b$ initio approach along with the "free" exciton. For defects in strong concentration, the coexistence of both bound and free excitons adds substructure to the main exciton peak and provides an explanation for the corresponding feature in cathodo- and photoluminescence spectra.
\end{abstract}

DOI: 10.1103/PhysRevB.83.144115

PACS number(s): 71.35.-y, 71.55.-i

\section{INTRODUCTION}

Boron-nitride crystals and nanostructures are excellent wide band-gap materials for the realization of ultraviolet (UV) lasers. ${ }^{1}$ Defects formed during the BN synthesis or introduced as impurities can bind excitons and act as centers for their recombination. This leads to (desired or undesired) luminescence bands and losses in the photon frequency range of interest. In fact, recent experiments $\mathrm{s}^{2-9}$ have given evidence that defects strongly effect the luminescence of BN-based materials. In order to achieve optimal optical properties, the relevant defects have to be identified such that methods can be devised to tune their concentration in the material.

Boron nitride (BN) is known to exist in three different crystals structures, namely hexagonal $\mathrm{BN}$ (h-BN), cubic $\mathrm{BN}$ (c-BN), and wurtzite $\mathrm{BN}$ (w-BN). Among these structures, $\mathrm{h}-\mathrm{BN}$ is the stable one at room temperature and ambient pressure. Like graphite, it consists of stacked BN layers with a honeycomb structure. BN nanostructures ${ }^{10,11}$ ranging from single and multiwall nanotubes to $\mathrm{BN}$ fullerens and nanocones can be envisaged as having been tailored from a single $\mathrm{BN}$ sheet. Unlike in graphite, the partially ionic character of the $\mathrm{BN}$ bond results in a wide band gap of about $6.5 \mathrm{eV}$ for bulk h-BN. ${ }^{12}$ The combination of a large gap with a strong electron-hole attraction makes the optical properties of $\mathrm{BN}$ nanostructures largely independent of the layer arrangement. ${ }^{11,13} \mathrm{~A}$ single layer of $\mathrm{h}-\mathrm{BN}$ is thus a generic system for studying the optical properties of crystalline and nanostructured BN including the exciton-defect interaction.

Detailed experiments on the cathodo- and photoluminescence of h-BN crystals and BN nanotubes have been performed in the recent years. ${ }^{2-9,14}$ The origin of some of the features in the BN luminescence spectra remains unsettled. For instance, the occurrence of a defect-related emission band at $4 \mathrm{eV}$, composed of regularly spaced peaks compatible with phonon replicas, ${ }^{5,7,15}$ can be associated with a deep level impurity. However, the defect that is responsible for this deep level has not yet been identified. Furthermore, the emission band at $5.77 \mathrm{eV}$ shows different subpeaks on the low energy side. The origin of its substructure is unclear, since the main peak can be understood as being due to the recombination of a Frenkel-type exciton ${ }^{12}$ with a degenerate recombination line. ${ }^{13,16}$ Interpretations of the experimental findings are based on different mechanisms: quasi-donor-acceptor pairs, ${ }^{6}$ excitons coupled with structural defects, ${ }^{9,15}$ breaking of the exact hexagonal symmetry (e.g., by the presence of defects), ${ }^{16}$ or a dynamical Jahn-Teller effect ${ }^{17}$ (symmetry breaking due to geometry relaxation in the excited state).

Theoretical works on defects in BN structures have so far focused on the electronic and structural properties of point defects. ${ }^{18-24}$ The abundance (related to the formation energy) and stability of different defects has been addressed with detailed density-functional theory (DFT) calculations..$^{20,25-29}$ The position of deep levels within the fundamental band gap has been calculated on the DFT level. However, DFT calculation suffer from the well-known underestimation of the band gap. Therefore, besides the neglect of the strong excitonic effects in BN, (de)excitation spectra based on DFT energy levels may not be reliable enough for comparison with experimental spectra.

In the present paper we address the optical properties of point defects in BN going beyond the approximation of DFT. We focus on a substitutional carbon impurity on the nitrogen site, the isolated vacancies and the $\mathrm{BN}$ divacancy that are common defects in BN. We will demonstrate that defects are responsible for the emission lines around $4 \mathrm{eV}$. Their presence can also explain the experimentally observed splitting 
of the main exciton peak. The paper is organized as follows. In Sec. II we summarize the computation methods employed in this work. In Sec. III we present the ground state results and the quasiparticle (QP) band structures. In Sec. IV we investigate the role of defects on the optical properties and finally in Sec. V we compare our results with experiments.

\section{COMPUTATIONAL METHODS}

Density functional theory (DFT) within the local spin density (LSDA) or gradient corrected approximations (GGA) for the exchange-correlation energy has been widely used to study defects in semiconductors and insulators. Although DFT is, in principle, exact, it is limited to the ground state and as a closed expression for the exchange-correlation energy functional is unknown, approximations like the LSDA or GGA have to be employed. Albeit theoretically well-founded, these approximate functionals bear inherent limitations like the well-known band gap problem which concomitantly affect the prediction of defect levels, in particular in wide band gap insulators. In the last years, many-body perturbation theory within the GW approximation ${ }^{30}$ has become a valid and accurate alternative to predict defects properties in semiconductors and insulators ${ }^{31-33}$ starting from the DFT ground state. Moreover, in going beyond the ground state, the optical properties of defects and the host insulator can be addressed by including the electron-hole interaction in the excited state via the Bethe-Salpeter equation (BSE). ${ }^{34-36}$

Here we use this approach to study the role of prototypical defects in the optical properties of $\mathrm{BN}$ nanostructures: a substitutional carbon impurity on the nitrogen sublattice $\left(\mathrm{C}_{\mathrm{N}}\right)$, the boron vacancy $\left(\mathrm{V}_{\mathrm{B}}\right)$, the nitrogen vacancy $\left(\mathrm{V}_{\mathrm{N}}\right)$, and the boron-nitrogen divacancy $\left(\mathrm{V}_{\mathrm{BN}}\right)$. Calculations proceed in three steps: first we obtain the DFT ground state employing the LSDA approximation and a plane wave pseudopotential method, ${ }^{37}$ (see Ref. 38 for details). Second we evaluate the quasiparticle band structure within the $\mathrm{G}_{0} \mathrm{~W}_{0}$ approach ${ }^{30,39,40}$ and third we address the optical spectra via the BSE. ${ }^{41}$ A very large supercell ${ }^{38}$ is employed to accommodate the defect and to enable at the same time the description of defect bound and free excitons. This becomes possible due to the strong localization of the bulk exciton state in $\mathrm{h}-\mathrm{BN},{ }^{12}$ in the layer, and tubes. ${ }^{13,42}$

As it has been shown that a large class of impurities, when introduced into h-BN, is in a spin-polarized state and acquires a local magnetic moment, ${ }^{24}$ we have performed spin-polarized calculations for the $\mathrm{C}_{\mathrm{N}}, \mathrm{V}_{\mathrm{B}}$, and $\mathrm{V}_{\mathrm{N}}$ cases while this was not necessary for the divacancy $\mathrm{V}_{\mathrm{BN}}$ that does not display a net spin polarization. The quasiparticle properties have been calculated starting from the Kohn-Sham Hamiltonian and orbitals within the $\mathrm{G}_{0} \mathrm{~W}_{0}$ approximation ${ }^{43}$ :

$$
E_{n k}=\epsilon_{n k}+Z_{n k} \operatorname{Re} \Delta \Sigma_{n k}\left(\epsilon_{n k}\right),
$$

where $\Delta \Sigma=\Sigma-V^{\mathrm{xc}}$ and $V^{\mathrm{xc}}$ is the LSDA exchangecorrelation functional, $\epsilon_{n k}$ are the Kohn-Sham eigenvalues, and $Z_{n k}=[1-\partial \operatorname{Re} \Delta \Sigma / \partial w]^{-1}$ is the renormalization factor. ${ }^{30}$ $\Sigma=\mathrm{GW}$, where the screened electron-electron interaction $W$ has been evaluated within the random-phase approximation (RPA) in terms of the dielectric function $\epsilon_{G, G^{\prime}}(q, \omega)$ using a plasmon-pole model. ${ }^{44}$ Neutral excitations are investigated on the basis of the quasiparticle spectrum by solving the Bethe-Salpeter equation (see Ref. 45 for details). Excitonic effects are analyzed through a comparison of the absorption spectra for independent particles with absorption spectra where electron-hole attraction is included on the level of the BSE.

As we are studying an isolated monolayer employing three-dimensional periodic boundary conditions, the size of the quasiparticle energies and the energy of the electron-hole attraction will depend on the intersheet distance. In Ref. 13 we had shown that the value of the gap of the pure h-BN sheet is $0.6 \mathrm{eV}$ higher for an intersheet distance is 80 a.u. than for an intersheet distance of 20 a.u. (value which we are using in the calculations presented here). At the same time we had observed an increase of the exciton binding energy with increasing intersheet distance that almost exactly cancels the variation of the band gap. We expect a similar cancellation effect in the presence of defects but cannot exclude a minor effect of the intersheet distance on the absolute position of defect peaks.

\section{DFT-GROUND STATE AND QUASIPARTICLE STATES}

In this section we address the electronic and geometric structure of the four defects and then compare the DFTelectronic structure with the quasiparticle states obtained from $\mathrm{G}_{0} \mathrm{~W}_{0}$ calculations.

In Fig. 1 the equilibrium geometry of the $C_{N}$ and the vacancies $V_{N}, V_{B}$, and $V_{B N}$ is shown along with the $B N$ bond
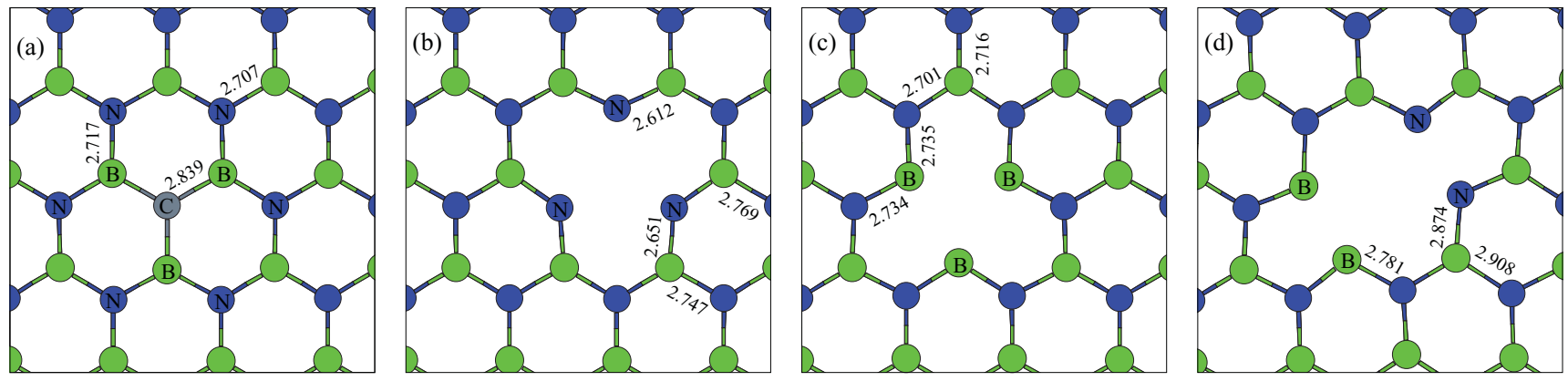

FIG. 1. (Color online) Geometry of the defects in BN monolayer with defects: (a) $C_{N}$; (b) $V_{B}$; (c) $V_{N}$; (d) $V_{B N}$. Defect induced changes of $\mathrm{BN}$ bond distances are indicated (in atomic units) and have to be compared with the BN distance of the perfect sheet (2.724 a.u.). 
distances in the vicinity of the defect center. In the case of substitutional impurity all the first neighbor B atoms of $C_{\mathrm{N}}$ undergo a small outward relaxation of 0.113 a.u. (4\% of the $\mathrm{BN}$-bond distance) with respect to the ideal lattice positions. The defect retains its threefold symmetry. In the case of $V_{B}$ and $\mathrm{V}_{\mathrm{N}}$ the outward displacement of the first-neighbor atoms is larger. More importantly, in agreement with Ref. 24, we find that a (pseudo) Jahn-Teller effect breaks the threefold symmetry slightly. In the divacancy $\mathrm{V}_{\mathrm{BN}}$ case, the threefold symmetry is already reduced to a mirror symmetry by the defect configuration itself. We observed that the first $\mathrm{N}$ and $\mathrm{B}$ neighbors decrease their mutual distance upon relaxation, as shown in Fig. 1(d). The defect geometries found here are in good agreement with those reported in Refs. 18 and 20.

As established already in earlier work, $\mathrm{C}_{\mathrm{N}}$ and the vacancies possess localized defect levels in the band gap. Here we briefly review the electronic structure of the defects as obtained in DFT-LSDA and then compare it with the quasiparticle states obtained on the level of the $\mathrm{G}_{0} \mathrm{~W}_{0}$ approximation where the DFT band gap error is corrected. Since the prediction of defect emission and absorption spectra hinges on the defect level positions, a correct prediction of these quantities is a crucial point for the investigation of optical properties.

The defect levels as obtained within the DFT-L(S)DA are reported in the left panel of Fig. 2. In the case of $\mathrm{C}_{\mathrm{N}}$ the defect levels arise from the three bonds of the substitutional carbon impurity with its boron neighbors. These bonds combine into two fully occupied states located within the valence band and a third half-occupied localized state within the band gap. As a result of the occupation with only one electron there is a spin-splitting of $\sim 0.5 \mathrm{eV}$ between the occupied (spin-up) and unoccupied (spin-down) state.

The situation is more complex for the boron vacancy. In this case a twofold degenerate and a nondegenerate defect state are formed from the unpaired orbitals at the three nitrogen neighbors. In agreement with Refs. 20, 26, and 29, these defect levels are located within the band gap. In the neutral vacancy the twofold degenerate level is occupied by three electrons and the nondegenerate level remains empty. Due to the Jahn-Teller distortion a small splitting of the degenerate level arises in addition to the exchange-splitting of the half- and unoccupied states. $\mathrm{V}_{\mathrm{B}}$ behaves as a triple acceptor.

For $\mathrm{V}_{\mathrm{N}}$ we obtain a half-filled level in the band gap, and two unoccupied levels almost degenerate in energy with the conduction bands. Due to spin-splitting their counterparts of opposite spin are found as resonances in the conduction band. Finally, in the case of the divacancy $\mathrm{V}_{\mathrm{BN}}$, there are four orbitals originating from the two boron and nitrogen neighbors. Only three are located in the band gap, one being occupied by two electrons and the upper two remaining empty.

The above picture obtained from the Kohn-Sham eigenvalues gives a qualitative description of the defect levels in the band gap. The position of the states is, however, quantitatively affected by the LSDA-band gap error and the approximate treatment of exchange with this XC functional as discussed above. Furthermore, the ordering of occupied and unoccupied states on the Kohn-Sham level may be different from that of the "true" quasiparticle energy levels (see, e.g., Ref. 30). The $\mathrm{G}_{0} \mathrm{~W}_{0}$ method yields quasiparticle energies and an improved description of exchange and screening effects. Due to the reduced dimensionality of the $\mathrm{BN}$ monolayer there is an incomplete screening of the Coulomb interaction, such that the quasiparticle corrections are expected to be quite large. We calculated the quasiparticle band structure for all the cases considered above. The calculated band gap is in agreement with the results of Ref. 13. In the right panel of Fig. 2 the position of the defect quasiparticle energies within the band gap is shown schematically. The $\mathrm{G}_{0} \mathrm{~W}_{0}$ corrections to the LSDA levels depend on the orbital occupation and on the wave-function character. ${ }^{30}$ The exchange splitting between occupied and unoccupied defect levels is strongly enhanced and the ordering of defect levels within the band gap is altered in some cases. In all cases we find that occupied levels are pushed closer to the valence bands and unoccupied ones closer to conduction bands. For $\mathrm{C}_{\mathrm{N}}$ the spin-splitting is significantly increased from $0.5 \mathrm{eV}$ to $3.1 \mathrm{eV}$. A large exchange splitting is also present in the case of $\mathrm{V}_{\mathrm{N}}$. For $\mathrm{V}_{\mathrm{B}}$ all unoccupied levels are pushed up by more then $2 \mathrm{eV}$, depending on the character of the wave function, and the level order is modified as well.
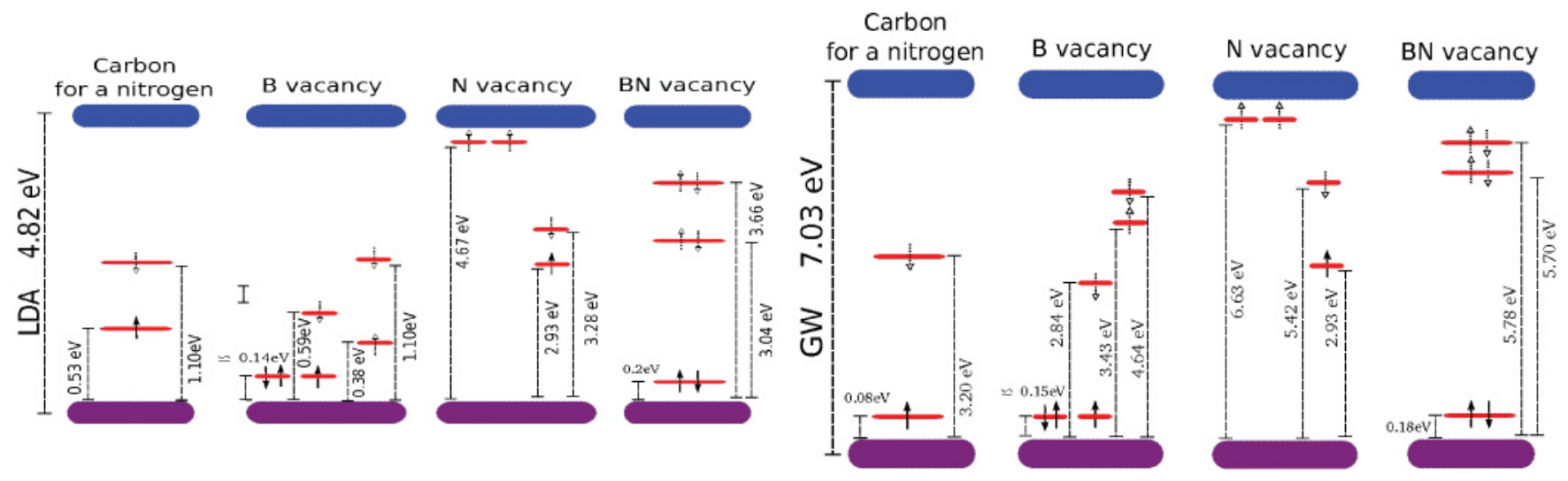

FIG. 2. (Color online) Quasiparticle energies of $C_{N}, V_{B}, V_{N}$, and $V_{B N}$ as obtained from LDA and $G_{0} W_{0}$ calculations: schematic representation of energy position with in the band gap. The filled arrows indicate levels occupied with electrons with spin up or down. Dotted arrows indicate unoccupied states (holes), correspondingly. 
In the case of $\mathrm{V}_{\mathrm{BN}}$, unoccupied levels are found close to the conduction band edge, while the occupied ones remain in their position close to the valence band edge. This underlines the fact that the divacancy contains both donor and acceptor levels. The general picture obtained from $\mathrm{G}_{0} \mathrm{~W}_{0}$ shows the clear deficiencies of the DFT-LSDA in the description of defect level positions in the case of BN, in particular, for unoccupied states. Vested with the defect quasiparticle spectrum we now turn to the optical properties.

\section{OPTICAL PROPERTIES}

In order to discern excitonic effects on the optical properties, we present first in Fig. 3 the optical spectra in the independent particle picture (IP) ${ }^{46}$ (i.e., without the inclusion of the electron-hole coupling). This yields an overview over relevant excitation mechanisms and later allows one to trace down the effect of the electron-hole coupling.

We start the discussion with the case of $\mathrm{C}_{\mathrm{N}}$. Comparing the corresponding panel of Fig. 3 with the schematic representation of the defect levels in the band gap (Fig. 2) one can identify the different absorption peaks. The first band at $3.4 \mathrm{eV}$ is due to transitions form the extended states at the valence edge to the defect levels within the band gap; the other band at $4.4 \mathrm{eV}$ involves extended states below the valence band edge and defect levels. Above $6 \mathrm{eV}$ we find the bulk-like spectrum that is only slightly perturbed by the presence of the defect. For the boron vacancy $\mathrm{V}_{\mathrm{B}}$ there are two bands at $3.5 \mathrm{eV}$ and $4.7 \mathrm{eV}$ that are associated with transitions from defect resonances in the valence band to the localized levels in the band gap and a series of small peaks around $5.9 \mathrm{eV}$ originating from valence band to defect transitions. The defect-related excitation bands of $\mathrm{V}_{\mathrm{N}}$ at $4.1 \mathrm{eV}$, in contrast to the previous cases, involves extended states near the conduction band edge as final states. Only the faint defect-related excitation band around $6.6 \mathrm{eV}$, not visible in the figure, originates from valance band states. Another faint peak around $5 \mathrm{eV}$ is due to transitions among the defect levels in the band gap. Finally, for the $V_{B N}$

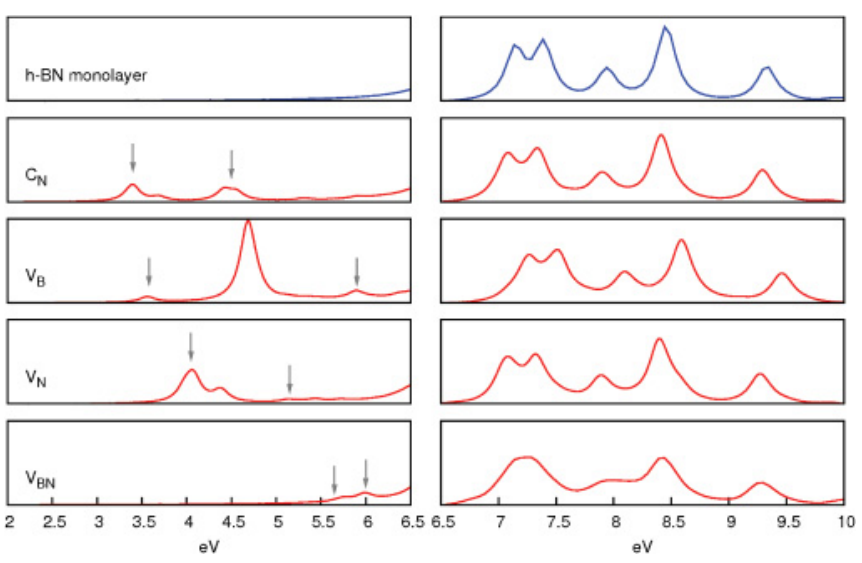

FIG. 3. (Color online) Independent-particle absorption spectra (arbitrary units) based on the $\mathrm{G}_{0} \mathrm{~W}_{0}$ quasiparticle band structure. In the left panel, the absorption cross section is increased by a factor 15 with respect to the right panel in order to make the defect-related peaks visible. divacancy, we find peaks between $5.6 \mathrm{eV}$ and $6.0 \mathrm{eV}$ due to defect-defect transitions and valence band to defect transitions, respectively.

We now turn to the spectra ${ }^{46}$ including the electron-hole interaction via the Bethe-Salpeter equation. These are shown in Fig. 4. The inclusion of the electron-hole interaction has essentially two effects on the defect-related spectra: (i) a general red-shift of the defect excitation energies due to electron-hole attraction and (ii) a coupling of the independent particle excitations. This coupling can give rise to bound electron-hole states, the excitons. In our case the BSE leads to a red-shift of defect-defect transitions in the range from 0.6 to $1.3 \mathrm{eV}$ depending on the character and the localization of the wave function. A common feature of all spectra is the large peak around $5.5 \mathrm{eV}$ which corresponds to the strongly localized exciton of pure $\mathrm{h}-\mathrm{BN}^{12,13,42}$ which is composed of a large number of independent electron-hole pairs. In the presence of a defect, electron-hole coupling leads to defect bound excitons besides the "free" excitons.

In the case of $\mathrm{C}_{\mathrm{N}}$ and $\mathrm{V}_{\mathrm{B}}$, as one can see from Fig. 4, an additional small peak appears just below the main excitons. In the case of $\mathrm{V}_{\mathrm{BN}}$ the main exciton band has three subpeaks due to the strong defect field with reduced symmetry. In order to disentangle the nature of the different peaks, we analyze the amplitude of the electron-hole pairs that compose each of the exciton eigenstates $\lambda$, as a function of the energy difference of the quasiparticle states of the corresponding electron and hole states:

$$
A^{\lambda}(\omega)=\sum_{\eta=\{e h\}}|\langle\eta \mid \lambda\rangle|^{2} \delta\left(\omega-E_{\eta}\right)
$$

For brevity, we report $A^{\lambda}(\omega)$ only for $\mathrm{V}_{\mathrm{B}}$ and $\mathrm{V}_{\mathrm{BN}}$ (see Fig. 5). The plots for the other defects show generally a similar behavior.

Comparing for $\mathrm{V}_{\mathrm{B}}$ the electron-hole amplitudes of the four excitation energies with the schematic diagram of Fig. 2, we can distinguish three kinds of excitations: (i) transitions from valence bands or defect resonances to the defect levels, that generate the peaks at $4.2 \mathrm{eV}$, (ii) the main exciton at $5.6 \mathrm{eV}$ that is entirely composed of extended states, and (iii) a peak at $5.4 \mathrm{eV}$ generated by the coupling of bulk excitations with transitions from and to defect levels in the band gap. The $\mathrm{C}_{\mathrm{N}}$ and $\mathrm{V}_{\mathrm{N}}$ cases present a structure similar to the one discussed above. From these results it is possible to infer three important consequences: (i) transitions from defect levels to valence or conduction bands with an energy close to the band gap energy mix with the bulk excitations giving rise to an additional peak close to the main exciton; (ii) transitions between defect levels are renormalized by the electron-hole interaction but do not mix with the bulk excitations; (iii) the main exciton peak maintains a structure similar to the bulk system.

The excitonic band of the divacancy $\mathrm{V}_{\mathrm{BN}}$ is distinct from the other spectra as the divacancy possess both acceptor and donor states of low symmetry in the vicinity of the band edges. The direct transitions between acceptor and donor states give rise to the absorption peaks around $4.8 \mathrm{eV}$, while the resonant coupling of transitions between the extended conduction and valence band states with donor and acceptor states splits the 

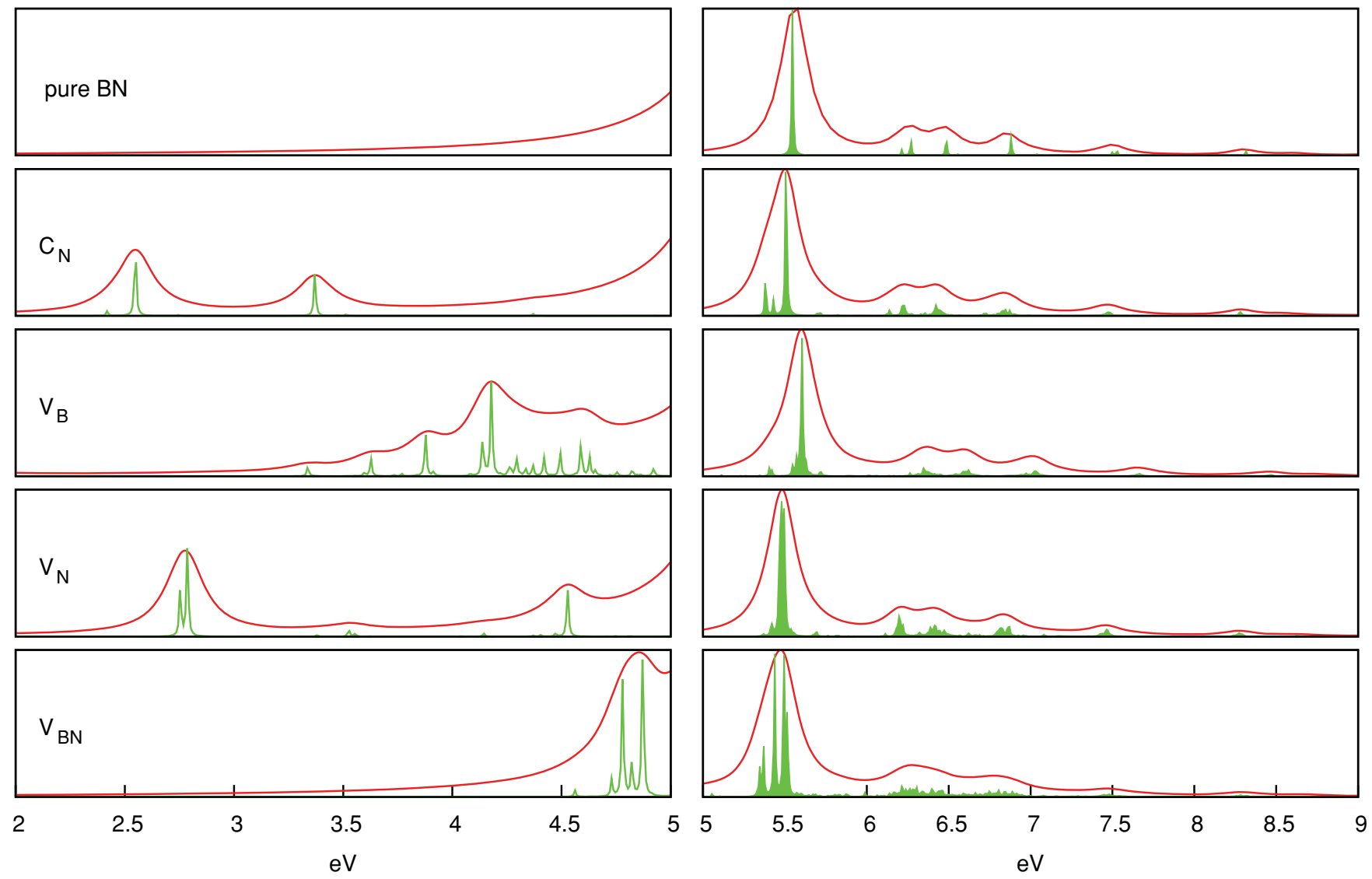

FIG. 4. (Color online) Optical absorption spectra calculated by the Bethe-Salpeter equation. In the left panels the absorption cross section is increased by a factor of ten in order to make the defect-related peaks visible. Spectra are shown with a broadening of $0.1 \mathrm{eV}$ (to simulate a typical experimental broadening) and $0.005 \mathrm{eV}$ (in order to investigate the fine structure of the spectrum).

main exciton in three subpeaks respectively at 5.37, 5.43, and $5.5 \mathrm{eV}$ (see Fig. 5).

The observed side peaks to the main excitonic peak are compatible with the fine structure observed in different luminescence experiments. ${ }^{5,6,15,17}$

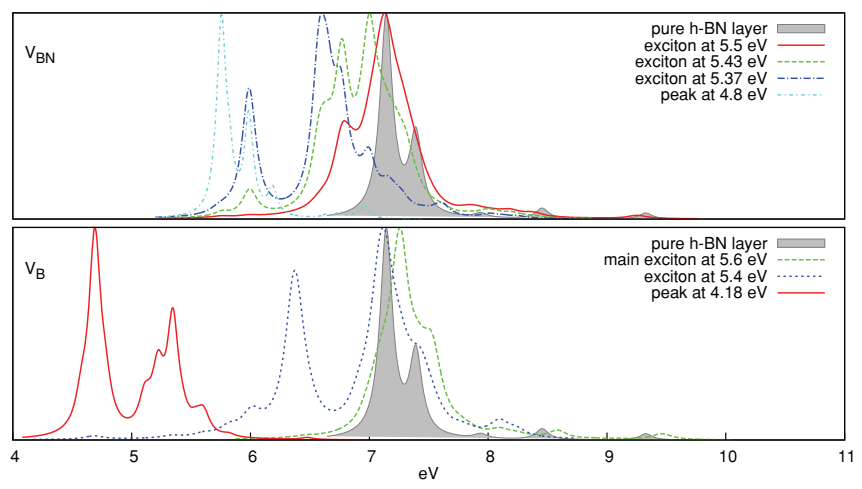

FIG. 5. (Color online) Amplitude of the different electron-hole pairing entering in the $\mathrm{BSE}$, for the boron vacancy and the $\mathrm{BN}$ divacancy, compared with the pure h-BN monolayer.

\section{CONCLUSION}

We have investigated the role of defects on the optical properties of $\mathrm{BN}$ nanostructures. We have treated four different plausible defects in a single sheet of hexagonal BN: a substitutional carbon impurity, a boron vacancy, a nitrogen vacancy, and a BN divacancy. Quasiparticle effects strongly modify the defect-level positions within the band gap and increase the exchange splitting between occupied and unoccupied levels. Electron-hole interaction (treated on the level of the Bethe-Salpeter equation) not only leads to a strongly bound excitonic state in pure $\mathrm{BN}$ but also strongly renormalizes transitions from and to defect levels. This means that the peaks in the optical spectra which are related to "deep level impurities" are strongly affected by the $e-e$ and $e-h$ interaction and can be reliably calculated only on the level of manybody perturbation theory (as opposed to the frequently used random-phase approximation using DFT wave functions and energies). Experimental luminescence spectra (as presented, e.g., in Ref. 15) display a prominent peak close to $4 \mathrm{eV}$ with phonon replica on the low energy side. Comparing the four theoretical absorption spectra (Fig. 4) with the experimental luminescence spectrum, the best agreement is obtained for the Boron vacancy where the main absorption peak of the low energy regime is at $4.2 \mathrm{eV}$. The Stokes shift due to possible relaxation of the excited state is not included in our calculation and limits the comparison of experimental luminescence with 
theoretical absorption spectra. In the case of a strong $(0.7 \mathrm{eV})$ Stokes shift, also the BN divacancy (where a defect related absorption peak is at $4.8 \mathrm{eV}$ ) could be responsible for the experimental observation. The nitrogen vacancy (absorption peak at $4.5 \mathrm{eV}$ ) might also explain the experiment. However, in this case a second strong luminescence peak should also be observable below $3 \mathrm{eV}$ which seems not to be the case in experiments.

The $10 \times 10$ supercell employed in our calculations is considerably larger than the extension of the wave function of the "free" exciton in pure h-BN. Therefore, besides the defect-related absorption peaks, our calculations also show the prominent absorption peak due to "free" excitons at $5.6 \mathrm{eV}$. This peak is doubly degenerate in the pure BN sheet. Coupling of the free exciton with one or several transitions from or to defect levels gives rise to a splitting of this peak and additional side peaks. The details of the splitting depend on the nature of the impurity but also on its periodic arrangement. ${ }^{47}$ The fine structure ${ }^{2}$ of the luminescence peak at $5.7 \mathrm{eV}$ (with additional side peaks at 5.46 and $5.64 \mathrm{eV}$ ) can possibly be explained through our calculations which clearly display defect-related side peaks of the "free" excitonic peak. Furthermore, any kind of breaking of the exact hexagonal symmetry can lead to a splitting of the bright doubly degenerate "free" excitonic peak into two peaks and will also (for bulk h-BN) render the "dark" exciton (located $0.1 \mathrm{eV}$ below the bright exciton) visible. ${ }^{16}$ For reasons of computational feasibility, we have performed calculations only on point defects. Other kinds of defects such as dislocations and grain boundaries lead similarly to a breaking of symmetry and/or to the formation of localized states and could thus as well explain the fine structure of luminescence spectra (one would expect a similar interaction of the "free" exciton with dangling-bond states). Indeed, Jaffrenou et al. had observed that the luminescence peaks at 5.46 and $5.64 \mathrm{eV}$ occur mostly at grain boundaries and dislocations ${ }^{15}$ where defect states due to dangling bonds are supposed to occur. The aim of our present study is thus not to unambiguously assign a certain type of defect to the observed luminescence spectra, but rather to show the general mechanism leading to the deep impurity levels and the formation of defect bound excitons. We also note that we did not study all possible point defects. A recent study of the chemical composition of an isolated h-BN sheet suggests the incorporation of oxygen atoms in the honeycomb lattice. ${ }^{48}$ The same study also demonstrated that substitutional carbon atoms occur mostly in pairs, occupying neighboring nitrogen and boron sites. Further studies are necessary to decide if the latter two types of defects can also occur during the synthesis of BN nanostructures or if they are due to the experimental conditions in the transmission electron microscope where vacancies in the $\mathrm{h}-\mathrm{BN}$ sheet are induced by the electron bombardment and may be subsequently filled by a substitutional atom.

An alternative route to symmetry breaking was proposed by Watanabe et al. ${ }^{17}$ They suggested that the dynamical JahnTeller effect (splitting of the degenerate exciton by polaronic effects) could explain the excitonic splitting in hexagonal BN. Further studies are necessary to disentangle the two effects.

\section{ACKNOWLEDGMENTS}

We acknowledge funding by the European Community through e-I3 ETSF project (Contract No. 211956). AR acknowledges funding by the Spanish MEC (FIS2007-65702C02-01), ACI-promciona project (ACI2009-1036), "Grupos Consolidados UPV/EHU del Gobierno Vasco" (IT-319-07). LW acknowledges funding by the French National Research Agency through Project No. ANR-09-BLAN-0421-01. This work was performed using HPC resources from GENCIIDRIS (Project No. 100063 and Project No. 091827). CA thanks X. Andrade and J. Alberdi Rodriguez for the efficient computer-cluster installation in San Sebastian. This work has been supported by Project No. FIS2010-21282-C02-01 (MCINN) from Spain.
${ }^{1}$ T. Taniguchi, K. Watanabe, Y. Kubota, and O. Tsuda, Production of a hexagonal boron nitride crystal body capable of emitting out ultraviolet radiation, (May 2010), patent no. US 2010/0120187 A1.

${ }^{2}$ K. Watanabe, T. Taniguchi, and H. Kanda, Nat. Mater. 3, 404 (2004).

${ }^{3}$ Y. Kubota, K. Watanabe, O. Tsuda, and T. Taniguchi, Science 317, 932 (2007).

${ }^{4}$ K. Watanabe, T. T. Takashi, and K. H. Kanda, Diam. Relat. Mater. 15, 1891 (2006).

${ }^{5}$ M. G. Silly, P. Jaffrennou, J. Barjon, J.-S. Lauret, F. Ducastelle, A. Loiseau, E. Obraztsova, B. Attal-Tretout, and E. Rosencher, Phys. Rev. B 75, 085205 (2007).

${ }^{6}$ L. Museur and A. Kanaev, J. Appl. Phys. 103, 103520 (2008).

${ }^{7}$ L. Museur, E. Feldbach, and A. Kanaev, Phys. Rev. B 78, 155204 (2008).

${ }^{8}$ P. Jaffrennou, F. Donatini, J. Barjon, J.-S. Lauret, A. Maguer, B. Attal-Tretout, and F. Ducastelle, Chem. Phys. Lett. 442, 372 (2007)
${ }^{9}$ P. Jaffrennou, J. Barjon, T. Schmid, L. Museur, A. Kanaev, J.-S. Lauret, C. Y. Zhi, C. Tang, Y. Bando, D. Golberg, B. Attal-Tretout, F. Ducastelle, and A. Loiseau, Phys. Rev. B 77, 235422 (2008).

${ }^{10}$ R. Arenal, X. Blase, and A. Loiseau, Adv. Phys. 59, 101 (2010).

${ }^{11}$ L. Wirtz and A. Rubio, in Optical and Vibrational Properties of Boron Nitride Nanotubes in B-C-N Nanotubes and Related Nanostructures, edited by Y. K. Yap, Lecture Notes in Nanoscale Science and Technology, Vol. 6 (Springer, Berlin, 2009).

${ }^{12}$ B. Arnaud, S. Lebègue, P. Rabiller, and M. Alouani, Phys. Rev. Lett. 96, 026402 (2006).

${ }^{13}$ L. Wirtz, A. Marini, and A. Rubio, Phys. Rev. Lett. 96, 126104 (2006).

${ }^{14}$ C. Zhi, Y. Bando, C. Tang, D. Golberg, R. Xie, and T. Sekigushi, Appl. Phys. Lett. 86, 213110 (2005).

${ }^{15}$ P. Jaffrennou, J. Barjon, J.-S. Lauret, B. Attal-Trétout, F. Ducastelle, and A. Loiseau, J. Appl. Phys. 102, 116102 (2007).

${ }^{16}$ L. Wirtz, A. Marini, M. Gruning, C. Attaccalite, G. Kresse, and A. Rubio, Phys. Rev. Lett. 100, 189701 (2008). 
${ }^{17}$ K. Watanabe and T. Taniguchi, Phys. Rev. B 79, 193104 (2009).

${ }^{18}$ S. Azevedo, J. R. Kaschny, C. M. C. de Castilho, and F. de Brito Mota, Eur. Phys. J. B 67, 507 (2009).

${ }^{19}$ T. M. Schmidt, R. J. Baierle, P. Piquini, and A. Fazzio, Phys. Rev. B 67, 113407 (2003).

${ }^{20}$ W. Orellana and H. Chacham, Phys. Rev. B 63, 125205 (2001).

${ }^{21}$ Z.-L. Hou, M.-S. Cao, J. Yuan, X.-Y. Fang, and X.-L. Shi, J. Appl. Phys. 105, 076103 (2009).

${ }^{22}$ F. Oba, A. Togo, I. Tanaka, K. Watanabe, and T. Taniguchi, Phys. Rev. B 81, 075125 (2010).

${ }^{23}$ M. S. Si and D. S. Xue, Phys. Rev. B 75, 193409 (2007).

${ }^{24}$ R.-F. Liu and C. Cheng, Phys. Rev. B 76, 014405 (2007).

${ }^{25}$ S. Okada, Phys. Rev. B 80, 161404 (2009).

${ }^{26}$ T. E. Mosuang and J. E. Lowther, Phys. Rev. B 66, 014112 (2002).

${ }^{27}$ L.-C. Yin, H.-M. Cheng, and R. Saito, Phys. Rev. B 81, 153407 (2010).

${ }^{28}$ A. Zobelli, C. P. Ewels, A. Gloter, and G. Seifert, Phys. Rev. B 75, 094104 (2007).

${ }^{29}$ A. Zobelli, C. P. Ewels, A. Gloter, G. Seifert, O. Stephan, S. Csillag, and C. Colliex, Nano Lett. 6, 1955 (2006).

${ }^{30}$ W. G. Aulbur, L. Jonsson, and J. W. Wilkins, in Solid State Physics, edited by H. Ehrenreich and F. Spaepen (Academic Press, New York, 1999), Vol. 54, p. 1.

${ }^{31}$ P. Rinke, A. Janotti, M. Scheffler, and C. G. Van de Walle, Phys. Rev. Lett. 102, 026402 (2009).

${ }^{32}$ S. Lany and A. Zunger, Phys. Rev. B 81, 113201 (2010).

${ }^{33}$ M. P. Surh, H. Chacham, and S. G. Louie, Phys. Rev. B 51, 7464 (1995).

${ }^{34}$ H. J. Mattausch, W. Hanke, and G. Strinati, Phys. Rev. B 27, 3735 (1983).

${ }^{35}$ Y. Ma and M. Rohlfing, Phys. Rev. B 77, 115118 (2008).

${ }^{36}$ M. Bockstedte, A. Marini, O. Pankratov, and A. Rubio, Phys. Rev. Lett. 105, 026401 (2010).

${ }^{37}$ P. Giannozzi et al., J. Phys. Condens. Matter 21, 395502 (2009).

${ }^{38}$ All DFT calculations have been performed using a $10 \times 10$ supercell containing 200 atoms. The distance between the BN sheet was 20 a.u. A single $k$ point was used. We used the exchange-correlation functional of Ref. 49. For the calculations, we used the PWSCF code $^{37}$ with Troullier-Martins pseudopotentials ${ }^{50}$ and a plane-wave cutoff of $50 \mathrm{Ry}$. All the atomic structures considered in the paper were relaxed using the Broyden-Fletcher-Goldfarb-Shanno (BFGS) algorithm and a convergence threshold for the forces of 0.0001 a.u. The $10 \times 10 k$-point sampling of the elementary cell (which corresponds to the $1 \times 1$ sampling of the $10 \times 10$ supercell) does not contain the high-symmetry point $K$, the point with the minimum direct gap. Therefore, the reported LDA gap of $4.82 \mathrm{eV}$ in Fig. 2 is slightly larger than the converged value $4.5 \mathrm{eV} .^{11}$

${ }^{39}$ G. Onida, L. Reining, and A. Rubio, Rev. Mod. Phys. 74, 601 (2002).

${ }^{40}$ M. S. Hybertsen and S. G. Louie, Phys. Rev. B 34, 5390 (1986).

${ }^{41}$ G. Strinati, Riv. Nuovo Cimento 11, 1 (1988).
${ }^{42}$ C.-H. Park, C. D. Spataru, and S. G. Louie, Phys. Rev. Lett. 96, 126105 (2006)

${ }^{43}$ Non-self-consistent GW calculations have been performed starting from DFT-LDA wave functions, using a plasmon pole approximation for the dielectric constant, ${ }^{44}$ with the code YAMBO. ${ }^{51}$ We use a single $k$ point, 3000 bands for expanding the Green's function, $40000 \mathrm{G}$ vectors for the exchange and 35000 for the correlation part of the self-energy. In contrast to Ref. 13 we did not add any scissor operator to correct the underestimation of the band gap in the $\mathrm{G}_{0} \mathrm{~W}_{0}$ approximation.

${ }^{44}$ The same dielectric constant has been employed in all the cases with and without defects, both for quasiparticle and Bethe-Salpeter equation. The dielectric constant has been calculated in the pure system, using 3000 bands, $3833 G$ vectors for the dielectric matrix, and $35000 G$ vectors for the wave functions entering in the calculation.

${ }^{45}$ The optical spectra have been calculated using the Bethe-Salpeter equation (BSE), ${ }^{41}$ with bands 300 to 500 (all $\pi$ and $\pi^{*}$ bands of the $10 \times 10$ supercell), a $10 \mathrm{eV}$ cutoff on the electron-hole pair energy entering in the BSE and $20000 \mathrm{G}$ vectors for the electronic wave functions.

${ }^{46}$ The independent-particle (IP) spectra are obtained for the $1 \times 1$ $k$-point set (corresponding to a $10 \times 10$ sampling of the elementary unit cell) and are not fully converged with respect to $k$-point sampling for transitions involving extended band states, despite the large supercells employed in the present calculations. Since we are only concerned with transitions involving localized defects levels, this is irrelevant for the analysis of excitation mechanism conducted here. Moreover we verified that peaks due to defects transition do not change increasing the number of $k$ points. In the BSE case, the main exciton collects most of the oscillator strengths. This speeds up the $k$-point convergence of the spectra and makes the presented spectra almost converged, contrary to the IP ones. In both cases, IP and BSE, we always considered the polarization parallel to the sheet.

${ }^{47}$ In our simulation, we use a 60 -degree unit cell which corresponds to a trigonal ordering of the defects. If a 90-degree supercell was chosen, the ordering of the defects would be rectangular. Only in the limit of very large unit cells, the splitting of the excitonic peak will be independent of the defect ordering and correspond to the coupling of a "free excitons" with an isolated defect. However, with the present computational resources, we cannot reach the limit of isolated impurities by increasing the supercell.

${ }^{48}$ O. Krivanek, M. Chisholm, V. Nicolosi, T. Pennycook, G. Corbin, N. Dellby, M. Murfitt, C. Own, Z. Szilagyi, M. Oxley, S. Pantelides, and S. Pennycook, Nature (London) 464, 571 (2010).

${ }^{49}$ D. M. Ceperley and B. J. Alder, Phys. Rev. Lett. 45, 566 (1980).

${ }^{50}$ N. Troullier and J. L. Martins, Phys. Rev. B 43, 1993 (1991).

${ }^{51}$ A. Marini, C. Hogan, M. Gruning, and D. Varsano, Comput. Phys. Commun. 180, 1392 (2009). 